\title{
Proyectos internacionales de bibliotecas virtuales en ciencias de la salud: características y buenas prácticas
}

\author{
International virtual library projects in Health Sciences: characteristics and good practices
}

\section{Montserrat Salas Valero (1), José Antonio Salvador Oliván (2)}

\author{
(1) Instituto Aragonés de Ciencias de la Salud. Avda San Juan Bosco, 13. 50009 Zaragoza, España; montse_salas@yahoo.es (2) \\ Departamento de Ciencias de la Documentación e Historia de la Ciencia. Universidad de Zaragoza. C/ Pedro Cerbuna, 12. 50009 \\ Zaragoza, España; jaso@unizar.es
}

\begin{abstract}
Resumen
Se analizan las características de un conjunto representativo de bibliotecas virtuales internacionales en ciencias de la salud y se identifican buenas prácticas que puedan extrapolarse a un modelo nacional en España. Para ello se seleccionaron proyectos de países con una gestión sanitaria descentralizada similar a la española. Se analizaron las variables: estructura, financiación, contenidos, coordinación de procesos y órganos de trabajo interbibliotecario; y se identificaron buenas prácticas que susceptibles de ser aplicadas a un modelo nacional, destacando la centralización de procesos y la presencia de órganos de cooperación interbibliotecaria.
\end{abstract}

Palabras clave: Bibliotecas virtuales. Ciencias de la salud. Buenas prácticas. Cooperación bibliotecaria. Redes de bibliotecas. Estudios comparativos.

\section{Introducción}

Las Bibliotecas Virtuales en Ciencias de la Salud (BVCS) pertenecen al grupo de bibliotecas especializadas que recogen, tratan y difunden información en el ámbito de ciencias de la salud. Además del acceso remoto a bases de datos, revistas y recursos de información electrónicos, deben ofrecer información de calidad a los profesionales de la salud para la toma de decisiones en su práctica clínica, investigación, docencia y gestión sanitaria (Canadian Health Library Association, 2008).

Entre las ventajas de estas bibliotecas destacan el ahorro de costes que supone para la organización a la que pertenecen; así, por ejemplo, se ha señalado en la literatura que el acceso a las fuentes de información tiene un impacto significativo en la toma de decisiones clínicas y reduce la frecuencia y severidad de los errores médicos (Marshall, 1992), reduce el tiempo de estancia del paciente en el hospital (Klein, 1994) y contribuye a una mejor atención al paciente (Health Educational England, 2015).

\begin{abstract}
The characteristics of a representative set of Health Sciences virtual libraries were analyzed with the further aim of identifying good practices that could be to put into practice in the Spanish context. Projects were selected in countries with a decentralized health management system similar the Spanish one. The following variables were analyzed: structure, financing, content, process coordination and interlibrary cooperation bodies. Also, good practices that can be applied to the Spanish national model were identified, of which we highlight the centralization of processes and the presence of organisms or interlibrary cooperation.
\end{abstract}

Keywords: Virtual libraries. Health sciences. Good practices. Interlibrary cooperation. Libraries network. Comparative studies.

En España no existe una Biblioteca Virtual (BV) para todo el Sistema Nacional de Salud, aunque ya a principios de los años 90 , dentro de las IV Jornadas Nacionales de Información y Documentación en Ciencias de la Salud, se planteara la necesidad de crear un sistema nacional de información y documentación que coordinase actividades en este ámbito (Rodríguez Alonso, 1993), cuestión recurrente en las siguientes ediciones y demandada por parte de los profesionales bibliotecarios. Con la culminación en el año 2002 del traspaso de las competencias en materia de salud a las comunidades autónomas (CCAA), y ante el aumento de precios de los recursos de información, las Consejerías de Salud de las CCAA comenzaron a apostar por la creación de BVCS regionales que posibilitaran un ahorro del gasto y proporcionaran a todos los profesionales sanitarios acceso a los mismos recursos independientemente de su lugar de trabajo, democratizando de esta manera el acceso a la información (Muñoz González y Juan Quilis, 2011).

Sin embargo, los problemas que impulsaron la creación de las BVCS autonómicas seguían exis- 
tiendo (aumento de costes, duplicidad de recursos), por lo que se consideró necesaria una actuación a nivel nacional. Y así, en 2008, el Ministerio de Sanidad, Servicios Sociales e Igualdad (MSSSI) puso en marcha un proyecto para estudiar la viabilidad de creación de una Biblioteca Virtual del Sistema Nacional de Salud; la coordinación técnica del proyecto recayó en la Biblioteca Virtual del Sistema Sanitario de Andalucía y participaron las 17 CCAA con un representante técnico. Las conclusiones del proyecto con la propuesta de un modelo se enviaron al Ministerio, pero nunca llegó a implantarse.

Posteriormente, en 2013, el MSSSI dió a conocer a los responsables de BV autonómicas la suscripción a Uptodate, una fuente de información clínica basada en la evidencia y diseñada para responder preguntas de manera rápida y concisa, y en octubre de 2014 adquirió una plataforma creada con la herramienta Discovery tool que permite recuperar en una única interfaz la información contenida en los recursos de cada comunidad autónoma, integrando todos los recursos digitales suscritos y gratuitos, así como los fondos de formato impreso de sus catálogos (González Guitián y Alonso, 2015). La propuesta del Ministerio tenía como objetivo desarrollar una estrategia única y transversal para todo el Sistema Nacional de Salud que coordinara los centros documentales y responsables de la gestión del conocimiento en las CCAA, desarrollar una plataforma de gestión del conocimiento que actuara como portal único de acceso a información científica proveniente de los principales proveedores de información (bases de datos, revistas, libros), facilitar a los profesionales sanitarios el acceso centralizado a recursos de información científica comunes para todo el SNS, diseñar una plataforma centralizada para CCAA, y dotar a la plataforma de una estructura orgánica y funcional que permitiera la incorporación y mantenimiento de recursos de información y servicios bibliotecarios.

El coste del proyecto fue asumido completamente por el Ministerio de Sanidad y la gestión recayó en la empresa Greendata. Consistía en la creación de un buscador para cada institución participante y un portal general con enlaces a cada una de las CCAA participantes; en septiembre de 2015 tan solo 3 comunidades autónomas (Canarias, Castilla y León, y Galicia) lo habían implantado completamente incluyéndolo en sus Bibliotecas Virtuales, no apareciendo en la web del Ministerio o de la Biblioteca Nacional de Ciencias de la Salud. Las razones de esta escasa implantación en las CCAA pudieron deberse a cuestiones políticas, técnicas o simplemente falta de interés.
La situación actual de España contrasta con algunos países en los que existe un modelo central de BVCS. Es por ello que el objetivo principal de este artículo es analizar las características principales de los modelos internacionales de Bibliotecas Virtuales de Ciencias de la Salud e identificar las buenas prácticas que puedan servir como fuente de referencia en la creación futura de un modelo integrador de las BVCS en España.

\section{Método}

En una primera fase se buscaron proyectos de Bibliotecas Virtuales en Ciencias de la Salud a nivel internacional. Para la selección de la muestra, se tomaron como referencia los datos ofrecidos por la OCDE, siendo España el país que en mayor medida ha descentralizado la toma de decisiones y la gestión en entidades subestatales (Joumard y otros, 2010). Se eligieron países con similar grado de descentralización que España, seleccionando los proyectos de Canadá, Finlandia, Noruega y Gran Bretaña. Además, debido a su relevancia, se seleccionó también el proyecto llevado a cabo en los EEUU (país no incluido en el estudio de la OCDE) y el de BIREME (como experiencia interestatal desarrollada en los países de Latinoamérica).

Las variables estudiadas, siguiendo el modelo utilizado por Roqué Castella (2011) en su estudio de redes de bibliotecas, fueron las siguientes:

- Estructura/Dependencia: Para conocer la dependencia institucional de la biblioteca, así como el tipo de organización y estructura de trabajo interna.

- Financiación: Información sobre las vías de financiación, con la finalidad de conocer el grado de apoyo institucional a nivel económico.

- Recursos/Contenidos: Incluye el análisis de los recursos que ofrecen de manera centralizada.

- Procesos coordinados/Centralizados: Se analizan los procesos que se realizan de manera centralizada y/o coordinada.

- Órganos formales de trabajo interbibliotecario: Para identificar la existencia de organismos (asociaciones, grupos de trabajo...etc.), que sirven de apoyo a los bibliotecarios que conforman la Biblioteca Virtual analizada.

La elección de estas variables se justifica porque abordan todos los aspectos necesarios para la interpretación de las funciones, objetivos, procedimientos y modos de trabajo, así como la identificación de los servicios prestados por cada uno 
de los proyectos, facilitando de esta manera un análisis comparativo posterior.

Para examinar las características de las BVCS, se utilizaron fuentes como documentos, memorias, páginas web e informes institucionales. También se realizó una entrevista por correo electrónico, en septiembre de 2015, a algunos responsables del proyecto de Canadá, concretamente a Jeanna Hough, presidenta de la Canadian Health Libraries Association y a Jim Henderson, investigador del Canadian Institutes of Health Research.

A partir del análisis de las variables anteriores, se identificaron buenas prácticas, término que se utiliza con frecuencia en áreas como la gestión sanitaria y el sistema educativo y que hace referencia a aquellas técnicas o metodologías que, a través de la experiencia y la investigación, han demostrado ser eficaces para conseguir un resultado deseado sin utilizar recursos excesivos y que ofrecen soluciones a problemas en otros contextos similares (Jerí Rodríguez, 2008).

\section{Descripción de los proyectos internacionales de BVCS}

\subsection{Canadá - Canadian Virtual Health Library $(\mathrm{CVHL})$}

La estructura territorial de Canadá y el nivel de desarrollo de sus bibliotecas regionales es muy similar a la organización administrativa y territorial de España. Este proyecto, tras los 3 años de vigencia de la subvención, finalizó en 2013 y en 2016 ya no existe ni siquiera el portal o página web de la biblioteca, resultando extremadamente complicado encontrar información sobre esta iniciativa nacional, por lo que se realizaron entrevistas a los responsables del proyecto. En dichas entrevistas se pudo conocer que el motivo que causó el cese de actividad fue el económico tras la finalización de la subvención por parte del CIHR. Aunque se presentó un informe de renovación del proyecto, no tuvo éxito ya que la crisis económica y la situación política no eran favorables.

\subsubsection{Estructura y dependencia}

Canadá es un país muy similar a España en cuanto a su grado de descentralización políticoadministrativa. Es una federación formada por diez provincias y tres territorios. Al igual que en España, las provincias disfrutan de un gran grado de autonomía en relación con el poder federal, y tienen la jurisdicción sobre sanidad, educación, bienestar social y transportes. Si bien el $70 \%$ de la sanidad en Canadá tiene financiación pública (federal y provincial), casi la totalidad de los servicios son provistos por el sector privado y la mayoría de los hospitales pertenecen a asociaciones sin ánimo de lucro.

Los médicos son autónomos y cobran de los gobiernos provinciales y territoriales por los servicios prestados. Pueden estar afiliados a organizaciones públicas o privadas, o actuar como docentes en los hospitales universitarios. La afiliación institucional del trabajador sanitario determina el servicio de biblioteca disponible y, por consiguiente, los recursos electrónicos a los que puede acceder. Según datos de la OCDE, en Canadá hay 2 médicos por cada 1000 habitantes (OECD, 2015).

La CVHL se creó para contribuir a mejorar la atención de la salud y promoción de la salud, facilitando a los profesionales sanitarios canadienses el acceso a la información para la toma de decisiones, desarrollo de políticas y programas, investigación, formación continua y desarrollo profesional, contribuyendo al funcionamiento eficaz y eficiente del sistema de atención de salud de Canadá y optimizando el uso de los recursos a través de la coordinación de los servicios existentes en todo el país (Canadian Health Library Association, 2008).

En cuanto a la dependencia, la Canadian Health Libraries Association (CHLA) fue quien promovió la iniciativa a la que se fueron sumando distintas organizaciones como hospitales, universidades, asociaciones de profesionales, autoridades sanitarias provinciales, instituciones científicas, etc., que propusieron conjuntamente la creación de esta biblioteca. En un exhaustivo informe evaluaron y analizaron el concepto de una Biblioteca Virtual en Salud (CVHL) en Canadá y estimaron la viabilidad y su potencial sostenibilidad a largo plazo.

El proyecto se basaba en los siguientes principios: a) el acceso equitativo a la información de salud de calidad garantiza una mejora de los cuidados, aumenta la seguridad del paciente y es una política pública esencial; b) las bibliotecas son componentes esenciales del proceso que incorpora nuevos conocimientos en la promoción de la salud y la práctica asistencial; y c) las bibliotecas locales son la base de una red canadiense de bibliotecas de salud.

Con el fin de promover el uso de información basada en la evidencia, se propuso aprovechar y potenciar los recursos de conocimiento disponibles a través de bibliotecas de salud canadienses, así como ofrecer servicios de intermediación bibliotecaria. 
Como objetivos estratégicos se incluían (Canadian Health Library Association, 2008): a) ampliar el acceso de los profesionales de la salud a nivel nacional a la mejor información en salud basada en la evidencia; b) acelerar la aplicación de los resultados de la investigación en la práctica asistencial y promoción de la salud, y c) aprovechar el poder de compra de los servicios de información sanitaria financiados con fondos públicos para maximizar el valor de los recursos.

En enero de 2010, comenzó a ponerse en marcha el proyecto. La primera actividad consistió en establecer redes y en la contratación de personal de base. Existía una oficina de coordinación nacional, con coordinadores locales y personal clave por áreas (Roqué Castella y otros, 2011).

En cuanto a las bibliotecas regionales, al igual que en España, algunas provincias tienen su propia red de bibliotecas en el ámbito de la salud o bien una Biblioteca Virtual central, y se pretendía que todas ellas se sumaran al proyecto de CVHL. Estos consorcios regionales, considerados en conjunto, juegan el papel de una red nacional, aunque de una manera un tanto irregular y poco sistemática.

\subsubsection{Financiación}

La CVHL fue financiada por el Canadian Institute of Health Research (CIHR) con 800.000 dólares canadienses durante el periodo 2009-2013, aunque no era una aportación para la suscripción y compra de contenidos sino para la puesta en marcha de una red canadiense de información en salud. Los objetivos de esta financiación eran crear un portal web y desarrollar una estrategia para aprovechar el poder de compra colectiva y maximizar el alcance e impacto de los recursos de información electrónicos de salud. La forma de pago de estos recursos era descentralizada, ya que los miembros del consorcio pagaban por los recursos a la CVHL en lugar de directamente a los proveedores, sin que ello implicara un aumento en las tarifas.

El proyecto iba dirigido a todo tipo de bibliotecas en el ámbito biomédico: hospitalarias, asociaciones, grupos de pacientes, universidades, escuelas, etc. La CVHL era considerada como una red de redes de bibliotecas en el que las éstas debían pagar una cuota por "suscribirse" al proyecto. Se le denominó "Red de Suscriptores". Estos suscriptores eran socios que podían participar en la compra colectiva de contenidos y servicios. Las suscripciones a través de la CVHL se extendían a los miembros del consorcio a través de sus consorcios locales, con lo que permitía que éstos mantuvieran su modelo de negocio existente. Los suscriptores también tenían la flexibilidad para decidir si querían adquirir la totalidad o parte de las colecciones o paquetes. Las cuotas por ser abonados consistían en el coste de los contenidos más un cargo administrativo para cubrir los costes de funcionamiento.

\subsubsection{Recursos y contenidos}

En cuanto al tipo de recursos, la CVHL contrató un conjunto básico de recursos comunes a los consorcios de información de salud: CINAHL, Cochrane/EBMR, Pyclnfo y STAT! Ref, a los que se accedía desde el portal mediante autentificación, aunque también estaban integrados los fondos de las bibliotecas participantes.

\subsection{4. Órganos formales de trabajo interbibliotecario}

La CHLA es la organización que desde el año 1975 aglutina a los bibliotecarios y especialistas en información de salud de Canadá, y la encargada de coordinar los diferentes proyectos puestos en marcha a nivel nacional. Actualmente trabaja en numerosos proyectos y su página web es el punto de encuentro de los profesionales en información en ciencias de la salud. Su misión es dirigir a los bibliotecarios y especialistas en información de salud hacia la excelencia a través del desarrollo profesional, la creación de redes y la promoción.

\subsection{Noruega - Norwegian Electronic Health Library (NEHL)}

\subsubsection{Estructura y dependencia}

Noruega es un país organizado en 19 provincias, con una media de 3,7 médicos por 1.000 habitantes (OECD, 2015). En cuanto a la estructura de la red sanitaria, el país se divide en 4 áreas de salud que incluyen centros de salud, clínicas y hospitales. Los centros de salud son responsabilidad de las provincias, que gestionan autónomamente el presupuesto sanitario. Los hospitales son públicos y las clínicas son fundamentalmente privadas, aunque con ayudas estatales, y en la práctica son sociedades de varios especialistas en diversas áreas que trabajan conjuntamente. La NEHL es una Biblioteca Virtual destinada a todos los profesionales y estudiantes de salud en Noruega. Fue puesta en marcha oficialmente el 6 de junio de 2006 por el Ministerio de Salud y depende del Norwegian Knowledge Centre for Health Services (Norwegian Electronic Health Library, 2014).

A nivel organizativo, la NEHL se define independiente, está dirigida por un editor jefe y se rige 
por unos estatutos propios. Un consejo, que representa a los organismos de financiación, asesora estratégicamente, y el consejo editorial, que representa a los usuarios y partes interesadas, asesora en cuanto a contenidos. Dispone de personal compuesto por profesionales de la salud, bibliotecarios, periodistas, editores web y administradores (Nylenna y otros, 2010).

\subsubsection{Financiación}

La NEHL está financiada públicamente a través del presupuesto nacional de Noruega y de las cuatro autoridades sanitarias regionales. Según sus datos, es una inversión rentable y eficiente, ya que han estimado que los contratos institucionales hubieran supuesto un coste tres o cuatro veces superior a la contratación pública nacional conjunta (Norwegian Electronic Health Library, 2014).

\subsubsection{Recursos y contenidos}

Ofrece un portal común con recursos entre los que destacan herramientas de información en el punto de atención al paciente (BMJ Best Practice y UpToDate), guías, bases de datos (Micromedex, PyscINFO, Cochrane Library, Guidelines International Network-GIN) y más de 3.000 revistas científicas a texto completo. Además, compila colecciones únicas de guías clínicas, revisiones sistemáticas y tests médicos en noruego (Tjensvoll, 2011). Por otra parte, en línea con el objetivo de acceso abierto, crearon el repositorio HeRA como archivo de trabajos de los hospitales, institutos de investigación y otras instituciones de salud noruegas no académicas.

El contenido está organizado en dos categorías principales: por tipo de información y por especialidades. Han desarrollado en cooperación con Vivisimo un sistema de búsqueda básica (más rápida y con acceso a recursos limitados) y una búsqueda general (más lenta y con acceso a más recursos) (Norwegian Electronic Health Library, 2014).

El acceso a todo el contenido para el personal sanitario se realiza por identificación de las direcciones IP institucionales o bien entrando con el equivalente al número de documento nacional de identidad. También ofrece información dirigida a los pacientes y al público, aunque en este caso tienen acceso a todo el contenido que no está limitado por los acuerdos bilingüe, permitiendo elegir a los usuarios una búsqueda por puntos de atención (más rápida y de suscripción, como las revistas científicas) siendo la política general proporcionar al público el máximo contenido posible (Tjensvoll, 2011).

\subsubsection{Procesos coordinados/centralizados}

Destaca la estrecha cooperación con los bibliotecarios, que participan en la selección de fuentes de información y que han sido capaces cambiar de un modelo de adquisición local a uno nacional con el objetivo de reducir costes. Además, realizan tareas de elaboración y mejora de las guías de usuario, así como las relacionadas con el márketing y difusión de la página web de la biblioteca electrónica.

\subsection{5. Órganos formales de trabajo interbibliotecario}

La colaboración interbibliotecaria se remonta a 1975 cuando los bibliotecarios de los hospitales crearon una sección de medicina dentro de la Asociación de Bibliotecas de Noruega, que ha hecho posible la colaboración entre bibliotecarios del campo de la medicina y la salud.

\subsection{Finlandia - FinElib y TerLib}

\subsubsection{Estructura y dependencia}

El responsable del sistema sanitario es el Ministerio de Asuntos Sociales y Salud, que se organiza a través de los Comités de Salud Municipales y los Consejos. Según datos de 2015, existen alrededor de 300 municipios, organizados en torno a 19 regiones, que tienen la responsabilidad de la promoción y prevención de la salud, la atención primaria, la rehabilitación y los servicios dentales. Según datos de la OCDE, hay 3,2 médicos por cada 1.000 habitantes (similar a la media de la CEE) y 21,7 enfermeras por cada 1.000 habitantes (el nivel más elevado de la CEE) (OECD, 2015).

FinELib, el proyecto nacional de biblioteca electrónica de ámbito interdisciplinar en Finlandia, fue lanzado por el Ministerio de Educación en 1997 con el objetivo principal de apoyar la educación superior, el aprendizaje y la investigación. Como objetivos secundarios se incluían aumentar la calidad de la información electrónica disponible para los usuarios, mejorar la recuperación de la información a través de Internet y desarrollar una interfaz para acceder a recursos de información (University of Helsinki, 2015). En el 2000, el programa fue asumido por la Biblioteca de la Universidad de Helsinki, que es también la Biblioteca Nacional de Finlandia.

Los miembros del Consorcio FinELib pueden participar en la Biblioteca Nacional a través de tres grupos diferentes: el grupo Directivo, el grupo Consorcio y el grupo de Expertos (Rajakili, 2002). 
El grupo Directivo es el responsable de la formulación de políticas y anualmente evalúa los resultados y trata los aspectos económicos. Está integrado por representantes de los máximos directivos de las universidades y bibliotecas de universidades, escuelas politécnicas, institutos de investigación, bibliotecas públicas, la Biblioteca Nacional y el Ministerio de Educación. Los asuntos más prácticos se discuten en el grupo Consorcio, cuyos miembros son directores de bibliotecas universitarias, escuelas politécnicas, bibliotecas de institutos de investigación y bibliotecas públicas. Este grupo es el encargado de preparar modelos de precios, negociar licencias y redactar planes de márketing, entre otros asuntos.

El grupo de Expertos, denominado TerELib, está formado por personal de las bibliotecas de las facultades de medicina, hospitales, escuelas politécnicas e institutos de investigación. Se ocupa de realizar un seguimiento del desarrollo de los recursos electrónicos y de hacer propuestas de contratación a FinELib. También evalúa materiales, prestando atención a los aspectos como el contenido, interfaz, orientación y coste-beneficio de los recursos. La Biblioteca Nacional de Ciencias de la Salud (Terkko) es la biblioteca de investigación de la Facultad de Medicina de la Universidad de Helsinki, y también funciona como una biblioteca científica para el Hospital Central de la Universidad de Helsinki ( $\mathrm{HUCH}$ ). Actúa también como una biblioteca nacional en medicina y ciencias de la salud, y ha sido designada como centro de servicio de información de la OMS en Finlandia (Terkko, 2015).

\subsubsection{Financiación}

La financiación es central a través del Ministerio de Educación, aunque existen compromisos de exigencia a las bibliotecas para que financien la adquisición de material electrónico. Las bibliotecas participan mediante una cuota que varía dependiendo del tipo de material. La cuota de financiación centralizada para los recursos generales y multidisciplinares es la más alta. Las universidades tienen que financiar únicamente una cuota del $20 \%$ del total. Para los recursos científicos específicos, por ejemplo los biomédicos, se requiere una participación del $50 \%$ de la financiación.

\subsubsection{Recursos y contenidos}

Según el último informe publicado por FinELib en 2012, se habían suscrito aproximadamente 38.000 revistas a texto completo, 112 bases de datos bibliográficas, 4.400 obras de referencia y 360.000 libros electrónicos. FinELib ha negociado, en nombre del consorcio, los acuerdos con
Elsevier Science Direct, Web of Science y Journal Citation Reports, aunque son las bibliotecas las que se hacen cargo del coste, es decir, sin financiación FinELib (University of Helsinki, 2015).

\subsubsection{Procesos coordinados/centralizados}

En general, las bibliotecas médicas han ganado mucho con esta iniciativa ya que se han visto obligadas a cancelar muchas de las suscripciones debido al aumento anual de los precios (Rajakiili, 2002). La Biblioteca Nacional de Ciencias de la Salud ha pagado el $50 \%$ de las bases de datos de Ovid y colecciones de texto completo, y FinELib el $80 \%$ de algunas colecciones de revistas como Academic Press y Springer. El apoyo financiero que estas bibliotecas reciben de FinELib es más del $25 \%$ del total de las adquisiciones electrónicas, sin tener en cuenta el ahorro de tiempo y costes por el hecho de adquirir de manera centralizada.

La concesión de licencias de recursos electrónicos y muchas de las tareas asociadas, por ejemplo, la recopilación de estadísticas de uso, catalogación de revistas, el desarrollo del portal y las encuestas a los usuarios, se realizan en la Biblioteca Nacional. El objetivo es proporcionar al consorcio un servicio integral en relación con la concesión de licencias y el uso de los recursos electrónicos.

\subsection{5. Órganos formales de trabajo interbibliotecario}

En Finlandia las bibliotecas se organizan en torno a un National Library Network Service, que ofrece apoyo a las bibliotecas del sistema: 17 universitarias, 25 politécnicas, 200 especializadas, 19 bibliotecas regionales públicas y más de 300 municipales. Como dijo su directora, Kristiina HormiaPoutanen (2011), "Small country, many possibilities for cooperation". Todas ellas tienen un consejo de cooperación y contribuyen de alguna manera al desarrollo de la red nacional de bibliotecas. En las reuniones conjuntas entre la Biblioteca Nacional y la red de bibliotecas, las bibliotecas están representadas por los presidentes de los consejos (Hormia-Poutanen, 2011).

La red nacional de bibliotecas gestiona de manera coordinada distintos servicios como un catálogo nacional e interdisciplinar, la coordinación de consorcios, la coordinación en la compra de hardware, software, licencias, el mantenimiento y desarrollo de software o el repositorio nacional. 


\subsection{Inglaterra - National Electronic} Library for Health (NeLH)

\subsubsection{Estructura y dependencia}

La NeLH surge de una estrategia del Departamento de Información de Salud de Inglaterra en la segunda mitad de la década de 1990, tomando como base las bibliotecas médicas del National Health Service (NHS) y con el objetivo de crear una biblioteca digital dirigida al público, pacientes y profesional sanitario que les proporcionara acceso a información sobre las mejores prácticas y últimos avances en el diagnóstico y tratamiento (Toth y otros, 2000). La NeLH se lanzó en noviembre de 2000, luego pasó a denominarse $\mathrm{Na}$ tional Library for Health, y en el año 2008 NHS Evidence, dependiendo del National Institute for Health and Care Excellence (NICE). EI NICE depende del Departamento de Salud, pero operativamente es independiente del Gobierno (National Health Service, 2014).

La estructura del NHS, en el momento de creación de la NLH, operaba en diferentes niveles. Hay que tener en cuenta que Gran Bretaña comparte con España un modelo basado en el Sistema Nacional de Salud, pero con financiación de los presupuestos generales del Estado. A nivel nacional, el Departamento de Salud determina la política y establece la dirección estratégica en nombre del Gobierno de turno, y también es el brazo ejecutivo del Servicio Nacional de Salud. El segundo nivel, el regional, puede ser considerado el más variado. Antes de la reforma del 2013, existían 130 fundaciones de atención primaria que controlaban el $80 \%$ del presupuesto del NHS y eran las responsables de asegurar que las necesidades de salud de la población estuvieran cubiertas. A nivel local existen fundaciones hospitalarias de agudos, salud mental, etc., que son independientes. Estos niveles se tuvieron en cuenta para establecer los servicios de la NLH. Por ejemplo hay algunos asuntos que se llevan a cabo a escala nacional, como la adquisición de revistas para el SNS o las cuestiones que tienen que ver con los derechos de autor y licencias. En el ámbito local recaen una serie de responsabilidades, particularmente relacionadas con la prestación de los servicios bibliotecarios.

La misión con la que nació la NeLH fue facilitar el acceso al conocimiento más actual y de calidad para mejorar la salud, la atención sanitaria del paciente y la práctica clínica, complementando los fondos y tareas de las bibliotecas regionales y locales que, a pesar de poseer competencias propias, trabajan en estrecha colaboración con la $\mathrm{NeLH}$. Su desarrollo se articuló en torno a 5 principios fundamentales (Gray y Lusignan, 1999):
(1) concentración en la calidad y no simplemente la cantidad de la información; (2) contenido: tanto "know how" como conocimiento; (3) abierta a los pacientes, médicos, público y administraciones; (4) formato electrónico, y (5) participación de los usuarios en la toma de decisiones.

En el Reino Unido cada región tiene su propio servicio nacional de salud, y en consecuencia, cada una de las regiones (Irlanda, Escocia y Gales) ha desarrollado diferentes bibliotecas electrónicas en ciencias de la salud que se describen a continuación brevemente.

En Irlanda del Norte, la denominada Medical \& HSC Library es la biblioteca central de salud, asistencia social y salud pública desarrollada por la Queen's University Medical \& HSC Library, en colaboración con el Departamento de Salud, Servicios Sociales y Seguridad Pública. Los servicios se ofrecen desde 6 bibliotecas principales en diferentes puntos de la provincia y a través de su portal "Honni", que es la principal puerta de entrada a los recursos electrónicos para todos los trabajadores del Departamento de Salud, Servicios Sociales y Seguridad Pública (médicos, enfermeras, matronas, farmacéuticos, dentistas, psicólogos, trabajadores sociales, gerentes...), con acceso a una amplia colección de recursos electrónicos: bases de datos, libros electrónicos y 1.000 revistas electrónicas. En cuanto a los servicios que ofrece, es posible solicitar en línea artículos de revistas, formación, asesoramiento en la búsqueda bibliográfica, préstamo de libros, renovación de libros en línea y la gestión de la cuenta personal de la biblioteca.

En Escocia, la llamada Knowledge Network integra toda una serie de recursos para la gestión del conocimiento en la práctica médica y facilita el acceso a portales, revistas, libros, información para pacientes, recursos de formación, herramientas web, etc. Ofrece más de 5.000 revistas suscritas para toda Escocia, 5.000 libros electrónicos y 80 bases de datos. Su página web se diferencia del resto porque está diseñada para que los propios usuarios la personalicen según sus necesidades de información, permitiendo crear espacios, mover paneles, activar RSS, etc. Destaca el desarrollo de un sistema de gestión de bibliotecas unificado, cuyo resultado ha sido el SHELCAT (Scottish HEalth Libraries CATalogue), que actúa como catálogo unificado de libros y que permite a los usuarios acceder y usarlo para el préstamo.

Y, por último, en Gales la e-Library NHS Wales, financiada por el Gobierno de Gales, es una biblioteca cuyo objetivo es facilitar el acceso a recursos de información relevantes que sirvan de soporte a la asistencia sanitaria. Se desarrolló 
dentro de la estrategia Informing Healthcare con el fin de modernizar las tecnologías de la información y explorar formas innovadoras de comunicación.

\subsubsection{Financiación}

La NeLH es un proyecto financiado por el Servicio Nacional de Salud en Inglaterra, pero las bibliotecas electrónicas en las tres regiones de Irlanda del Norte, Escocia y Gales, son financiadas por sus propios sistemas sanitarios.

En junio de 2004, la NHS Information Authority con el apoyo de la NLH, encargó un estudio sobre la financiación de las bibliotecas del NHS. EI informe señaló que el paisaje de las bibliotecas se había modificado y modernizado, con un fuerte énfasis en la práctica basada en la evidencia y cada vez más centradas en el paciente. También destacó que, tras la revisión de las actividades de la administración pública en el Reino Unido, se había establecido para el Departamento de Salud un ahorro del $2,7 \%$ anual ( $£ 6.470$ millones en 2007-8) (Hill, 2008). Es importante resaltar que el informe identificó el gasto anual en servicios de biblioteca NHS en torno a los $50 \mathrm{mi}-$ llones de libras, lo que se consideraba innecesariamente complejo e insostenible.

\subsubsection{Recursos y contenidos}

La NeLH tenía como rol central ofrecer a los profesionales del NHS una colección de revistas, publicaciones secundarias, así como acceso a bases de datos como la Cochrane o Clinical Evidence. Uno de los proyectos más interesantes son las Virtual Branch Libraries, que cumplen con el objetivo de crear portales temáticos basados en la evidencia científica. En 2003, 19 bibliotecas especializadas fueron las encargadas de su desarrollo comenzando a cubrir temas como el cáncer, urgencias, atención primaria, genética, salud mental, pediatría, enfermedades infecciosas, diabetes, etc.

Otros de sus proyectos fue Professional Portals, que ofrecían información extraída de la $\mathrm{NeLH}$ y de las Virtual Branch Libraries destinadas a grupos específicos de profesionales: médicos de atención primaria, dietistas, personal de enfermería, terapeutas ocupacionales, fisioterapeutas, podólogos, etc.

\subsection{4. Órganos formales de trabajo interbibliotecario}

Mientras que todas las bibliotecas regionales ofrecen acceso a recursos de información, cada una difiere en su alcance y ámbito de competen- cias. La coordinación de esta red está centralizada en el NHS y recae en un equipo de bibliotecarios distribuidos en tres grupos de trabajo: a) desarrollo de contenidos; b) servicios a los socios de la $\mathrm{NeLH}$, que se ocupa de que los recursos suscritos sean accesibles para todo el sistema, y c) cooperación en grandes proyectos relacionados con el diseño y arquitectura de la web.

La comunicación de la NeLH con los responsables de las bibliotecas regionales es fluida gracias a la creación de un grupo de trabajo de bibliotecas regionales. La NeLH está integrada por más de quinientas bibliotecas del NHS, además de algunas universidades y bibliotecas de organismos independientes.

Con el fin de reunir a los profesionales de las bibliotecas y trabajar de forma integrada y coordinada, en 2003 se desarrolló una red de bibliotecas digitales Al participar en esta red, los bibliotecarios se beneficiaban del apoyo y de la formación y desarrollo profesional, al tiempo que ofrecía oportunidades para integrar las iniciativas nacionales y locales. La red creó un blog colaborativo para facilitar el intercambio de buenas prácticas e ideas.

Por otro lado, el National Knowledge Service (NKS) es una iniciativa que dispone de una estructura en red para proporcionar la mejor evidencia actual para el trabajo de la Biblioteca Virtual y del NICE. Entre sus objetivos destacan (Health Education England, 2015): a) llevar a cabo actividades que se benefician de las economías de escala, incluida la gestión de un conjunto de recursos electrónicos accesibles a todo el personal de atención sanitaria y social; b) minimizar las desigualdades de servicio; c) proporcionar oportunidades de desarrollo personal entre los programas locales y nacionales del NHS; d) promover la innovación en la biblioteca; e) adoptar y difundir buenas prácticas en los servicios bibliotecarios en todo el NHS, y f) participar con otras bibliotecas de salud externas al NHS.

Recientemente se ha publicado un marco estratégico para el desarrollo de la biblioteca y el Knowledge Service de NHS Inglaterra, 2014-2028, con una inversión total de 69 millones de euros para financiar la mayor parte de las fuentes de información.

\subsection{Estados Unidos - National Library of Medicine (NLM)}

\subsubsection{Estructura y dependencia.}

EEUU cuenta con un sistema sanitario complejo y con 2,4 médicos por cada 1000 habitantes (OECD, 2015). La NLM forma parte del Instituto 
Nacional de Salud que, a su vez, pertenece al Departamento de Salud de los EEUU, y es uno de los modelos de referencia en el ámbito de la información en ciencias de la salud. El desequilibrio entre los recursos de las bibliotecas médicas y las necesidades de información de los profesionales de la salud llevó en 1965 a la NLM a iniciar el desarrollo de una red de bibliotecas médicas, estableciendo bibliotecas médicas regionales que vincularan a la NLM con las instituciones locales. Este empuje hacia la normalización de la biblioteca se produjo gracias al trabajo conjunto de documentalistas y bibliotecarios médicos asociados a la Medical Library Association (MLA) y la Association of American Medical Colleges (AAMC).

A nivel legislativo, desde sus inicios ha estado dotada de contenido y estructura. Sus principales componentes incluyen: a) aspectos operativos de la biblioteca: adquisición, catalogación, indización, servicio de referencia, preservación, préstamo interbibliotecario, gestión de base de datos en línea, información sobre investigación y coordinación de la Red Nacional de Bibliotecas de Medicina; b) programas extramurales: gestión de las becas para proyectos de investigación y la formación en investigación en informática/información biomédica; c) servicios especializados de información, como toxicología y salud ambiental; d) Centro Nacional de Comunicaciones Biomédicas Lister Hill; e) Centro Nacional de Información Biotecnológica (NCBI), y f) Oficina de Informática y Sistemas de Comunicación.

\subsubsection{Financiación}

La fuente de financiación es eminentemente pública, del gobierno federal. En 2014 fue de 382 millones de dólares (National Institutes of Health, 2015).

\subsubsection{Recursos y contenidos}

Esta iniciativa supuso el inicio de una colaboración que favoreció la creación de herramientas de cooperación interbibliotecaria (catálogo colectivo de publicaciones periódicas) o sistemas para compartir los archivos de MEDLARS (MEDline And Retrieval System), hasta llegar una década después a prestar servicios de búsquedas bibliográficas a bibliotecas y usuarios, e implementar el servicio de obtención de documentos. En la actualidad produce distintas bases de datos como Medline, MedlinePlus, ToxNet o ClinicalTrials.gov. También destaca Pubmed Central, como archivo de revistas a texto completo y de libre acceso.

\subsubsection{Procesos coordinados/centralizados}

Destaca el préstamo interbibliotecario, a través del sistema DOCLINE, en el que participan las más de 6.000 bibliotecas que forman parte de la red. También ha desarrollado un software denominado Loansome Doc, para que las bibliotecas gestionen la petición de documentos con sus usuarios.

\subsection{5. Órganos formales de trabajo interbibliotecario}

La AAMC puso en marcha un programa regional de bibliotecas médicas mediante el que creaba una red nacional de bibliotecas médicas, que posteriormente pasó a llamarse National Network of Libraries of Medicine (NN/LM), de estructura piramidal, formada por ocho bibliotecas regionales coordinadoras de servicios en áreas que abarcan diferentes Estados, con el fin de que las bibliotecas miembros y centros de información integrantes de esta red proporcionaran a los profesionales de la salud y al público en general, recursos y servicios de información sanitaria ( $\mathrm{Na}$ tional Library of Medicine, 2015).

La coordinación de esas bibliotecas se lleva a cabo por la NN/LM. Según datos extraídos de su página web, en marzo de 2016 la red está formada por 6.487 organizaciones, de las cuales 125 son bibliotecas de universidades o del ámbito académico y más de 5.000 son bibliotecas principalmente de hospitales y clínicas.

Hay dos categorías de miembros en el NN/LM: miembro titular y miembro afiliado. Un miembro titular puede ser cualquier biblioteca o centro de información relacionado con las ciencias de la salud y que disponga de conexión a Internet, tenga su propia colección (libros, revistas, audiovisuales, bases de datos electrónicas), proporcione servicios de información dirigidos a profesionales de la salud y/o el público en general y participe en el programa de préstamo DOCLINE. Un miembro afiliado es toda biblioteca, centro de información u organización que promueva la información sobre la salud, pero que no cumpla con todos los criterios exigidos para ser miembros titulares.

Las condiciones que se exigen a ambos miembros para formar parte de la red, son los siguientes: a) designar a una persona de contacto local para la red de información/comunicación; b) estar en el directorio nacional de miembros de la red; $y$ c) proporcionar información actualizada sobre sus servicios y/o colecciones a la NLM y a su biblioteca regional de medicina. 
Estados Unidos, a través de su NLM, respeta la autonomía de cada centro y Estado en la adquisición de recursos, buscando un objetivo común a todas las bibliotecas médicas: el desarrollo de una base de datos del conocimiento, logrando un efectivo trabajo en red mediante la National Network of Libraries of Medicine.

\subsection{Biblioteca Virtual en Salud - BIREME}

Este caso no forma parte de un sistema sanitario nacional sino que integra una red de Bibliotecas Virtuales que tiene por objeto la cooperación a nivel técnico en información en ciencias de la salud con el fin de dar respuesta a las necesidades de los países de América Latina y del Caribe en cuanto a la producción y difusión de fuentes de información en salud en Internet.

\subsubsection{Estructura y dependencia}

Creada en Brasil en 1967, con el nombre de BIblioteca REgional de MEdicina (BIREME), tuvo como misión atender la demanda de literatura científica actual por parte de los sistemas nacionales de salud y las comunidades de investigadores, profesionales y estudiantes. Posteriormente, en 1982, pasó a llamarse Centro Latinoamericano y del Caribe de Información en Ciencias de la Salud (Packer, 2000). Su modo de trabajo es la colaboración en red. Cada país tiene un sistema nacional de información en ciencias de la salud que actúa como representante en el desarrollo de las políticas que propone BIREME. Según su última memoria publicada, "la red Biblioteca Virtual en Salud (BVS) está orientada a crear, fortalecer y desarrollar capacidades e infraestructuras nacionales de información técnica y científica, con el objetivo de promover acceso equitativo al conocimiento y evidencias científicas actualizadas" (Bireme, 2011).

BIREME se estructura de la siguiente manera: a) Estados miembros: todos los Estados de la Organización Panamericana de Salud (OPS); b) Estados participantes: Estados miembros de la Organización Mundial de la Salud (OMS) y que no son miembros de la OPS; y c) Organizaciones participantes: organizaciones internacionales públicas con experiencia en el campo de la información y la comunicación científico-técnica.

También hay un Comité Asesor, formado por la OPS y Brasil como miembros permanentes, y otros cinco Estados miembros de la OPS seleccionados por el Consejo Director de la Organización. El Comité tiene un mandato de tres años y sus funciones incluyen hacer recomendaciones al Director de la OPS sobre funciones programáticas, plan de trabajo y financiación.
El Comité Científico está compuesto por cinco miembros especialistas propuestos por los Estados miembros de la OPS y por la Directora de la OPS, y nombrados por el Comité Asesor. Su función es asesorar al Director de la OPS y al Comité Asesor de BIREME en la preparación e implementación de planes de trabajo, recomendar la adopción de nuevos productos, etc.

Por último, hay una Secretaría, órgano permanente, formado por el Director de BIREME y por un equipo técnico-administrativo. Es responsable de la gestión y ejecución de las funciones de BIREME.

\subsubsection{Financiación}

La red BIREME es financiada por la OPS y, en parte, también por el Gobierno de Brasil. Además reciben subvenciones con regularidad y cuenta con la colaboración de la Secretaría de Salud del Estado de São Paulo y Universidad Federal de São Paulo.

\subsubsection{Recursos y contenidos}

Los recursos de información que ofrece el portal de BIREME son, en su mayoría, bases de datos como la Literatura Latinoamericana y del Caribe en Ciencias de la Salud (LILACS), Medline, Biblioteca Cochrane Plus, literatura en casos de desastres de la OPS, Índice Bibliográfico Español en Ciencias de la Salud (IBECS), Literatura de la Biblioteca de la sede de la OPS, Literatura de Ingeniería sanitaria y ciencias del ambiente (REPIDISCA), Publicaciones de la OMS (WHOLIS), Campus Virtual de Salud Pública, y un Localizador de información en la salud.

En cuanto a los servicios que ofrece destaca el Servicio Cooperativo de Acceso a Documentos (SCAD), que facilita el acceso a documentos del área de ciencias de la salud exclusivamente para fines académicos y de investigación. Es un servicio en cooperación con las bibliotecas de la Red BVS en América Latina y el Caribe, y requiere pago por el coste de reproducción y envío de los documentos. Está disponible solamente para usuarios o instituciones registradas (bibliotecas, centros de información, etc.), por lo que los usuarios individuales pueden hacer uso del SCAD a través de una Biblioteca de la Red en sus países.

\subsubsection{Procesos coordinados/centralizados}

De manera coordinada, BIREME ha conseguido que todos los países miembros posean la misma estructura web para su BV nacional, y ha conseguido formar grupos de trabajo estables para el desarrollo y mantenimiento de sus diferentes productos, compartiendo una misma metodología 
para la producción de fuentes de información locales.

\subsection{5. Órganos formales de trabajo interbibliotecario}

El progreso de las actividades de BIREME se evidencia a través de la cooperación técnica de la BVS y redes asociadas, que está presente en 31 países e involucra a más de 2.000 instituciones. En marzo de 2016, la BVS cumplió 18 años de actividad, y ha logrado a través de sus productos y servicios una democratización del acceso, publicación y uso de la información científica en salud en esa área geográfica.

Consolidada como estrategia de cooperación técnica en información científica en salud en la región de la América Latina y Caribe y extensible para otras regiones en desarrollo, la BVS es promovida y coordinada por la Organización Panamericana de Salud / Organización Mundial de la Salud por medio del Centro Latinoamericano y del Caribe de Información en Ciencias de la Salud (BIREME/OPS/OMS).

\section{Conclusiones}

Se han identificado y analizado las características principales de BVCS a nivel internacional en cuanto a estructura, financiación, procesos coordinados y órganos formales de trabajo interbibliotecario.

Todas las bibliotecas virtuales analizadas poseen una estructura en el ámbito de la gestión sanitaria muy descentralizada, lo que sin duda es un factor muy importante para el desarrollo y viabilidad de sus proyectos y para el planteamiento de políticas y estrategias nacionales de documentación en ciencias de la salud.

Ha quedado plasmada la similitud entre todas ellas en cuanto al objetivo por el que se crearon y sus principales funciones; sin embargo todas difieren en algún punto en relación con su estructura, organización, financiación y gestión. En cuanto a la dependencia, los proyectos de Noruega y Finlandia dependen directamente de ministerios, sin embargo en Inglaterra y en EEUU dependen de los servicios públicos de Salud, aunque la gestión en todos ellos es realizada por organismos dependientes de éstos. La estructura en la mayoría de los casos es en red (Canadá, Inglaterra, EEUU, BIREME).

Todas producen beneficios al sistema nacional de salud y, por ello, se han sido los gobiernos centrales los que han apostado por su desarrollo. La financiación es pública en todas, con asignaciones directas de las instituciones sanitarias correspondientes, aunque en el caso de Finlandia están muy claros los co-pagos que deben hacer las instituciones participantes en la red nacional.

Existen importantes diferencias en los servicios y contenidos que debe ofrecer una iniciativa nacional: unas apuestan por el control de la producción científica de la región mediante repositorios, otras por la compra centralizada de revistas electrónicas (Canadá, Noruega, Finlandia, Inglaterra) y otras por la creación de bases de datos o de productos propios, ofreciendo información al paciente (Inglaterra, Noruega, BIREME y EEUU), pero todas comparten un objetivo común: mejorar la salud de la ciudadanía mediante el acceso de todos los profesionales de salud a la mejor información biomédica que les permita tomar decisiones basadas en la evidencia.

Las buenas prácticas identificadas giran en torno a la centralización de suscripciones y de procesos, demostrando ser la mejor herramienta para facilitar el acceso a recursos de información de calidad a todos los niveles (asistencial, investigador, formación y de gestión). Se ha destacado también como como buena práctica la presencia de un organismo o asociación de bibliotecas en CS que favorece la colaboración entre ellas. Asimismo el hecho de presentar la información por temáticas, resulta de gran utilidad para los profesionales sanitarios.

Estas prácticas han demostrado ser efectivas, pueden ser transferibles y representan un elemento innovador. Uno de los mayores motivos es el relacionado con la reducción de costes tanto en la adquisición de recursos como en la prestación de los servicios, unido a una mayor eficacia en su prestación derivada de la coordinación e implicación del personal disperso en los centros de atención sanitaria.

En el campo de las bibliotecas en ciencias de la salud, España está por detrás de otros países con un similar nivel de descentralización de competencias en el ámbito sanitario y carece de una estrategia nacional que corrija las disparidades e inequidades en cuanto al servicio y el acceso a recursos de información para los profesionales de salud y que ofrezca respuestas a las demandas de éstos. La cooperación a nivel nacional en modo de red de redes de bibliotecas puede optimizar el gasto en recursos bibliográficos y de gestión documental, lo que daría como resultado una racionalización y una homogenización del acceso a fuentes de información científica y que finalmente facilitaría la consecución de la equidad.

La creación de una Red Nacional de Bibliotecas Virtuales en Ciencias de la Salud, resulta de alto interés y está justificada desde diferentes puntos 
de vista. En primer lugar, la equidad en la prestación de servicios de salud para todos los grupos de población en todo el territorio nacional implica la igualdad de acceso a los conocimientos dentro del sistema de salud. En estos últimos años, se ha recortado el presupuesto destinado a bibliotecas, sin embargo se ha incrementado la presión a los profesionales médicos para que se mantenga la calidad asistencial con un uso más efectivo y racional de los recursos sanitarios.

En segundo lugar, los profesionales sanitarios requieren información de alta calidad basada en la evidencia científica, por lo que los recursos de una biblioteca a nivel nacional deben de ser evaluados por personal experto en documentación científica para seleccionar aquellos que aporten calidad (por encima de la cantidad).

\section{Referencias}

BIREME/OPAS/OMS (2011). Guía de la BVS 2011. São Paulo: BIREME/OPAS/OMS

Canadian Health Library Association (2008). Canadian Virtual Library - Feasibility study and readiness assessment. Canada Health Infoway Phase 0 Report. http://chlaabsc.ca/nnlh/cvhl-feas.pdf (2015-10-22).

Dalhousie University (2015). Atlantic Health Knowledge Partnership. http://tinyrul.com/pyny2od (2015-10-22).

DiCenso, A.; Bayley, L.; Haynes, R. B. (2009). Accessing preappraised evidence: fine-tuning the $5 \mathrm{~S}$ model into a $6 \mathrm{~S}$ model // Evidence Based Nursing. 12:4 99-101.

González Guitián, Carlos; Alonso, María Luisa (2015). Biblliotecas virutales de ciencias de la salud: realidad y oportunidad // Atención Primaria. 47: 5 264-266

Gray, J. M.; de Lusignan, S. (1999). National electronic library for health (NeLH) // British Medical Journal. 319:7223 1476-1479.

Health Education England. NHS (2015). Developing people for health and healthcare Knowledge for healthcare: a development framework for NHS library and knowledge services in England Preface to the Framework. For NHS library and knowledge services in England 2015-2020. http://tinyurl.com/z4bz9zv (2015-6-21)

Hill, P. (2008). Report of a national review of NHS health library services in England: from Knowledge to health in the 21st Century. http://tinyurl.com/d4pr9vk (2015-4-12)

Hormia-Poutanen, K. (2011). National Library and Library Network in Finland: cooperation being the driving force of success. (2015-4-21)

Jerí Rodríguez, Doris. (2008). Buenas prácticas en el ámbito educativo y su orientación a la gestión del conocimiento. // Educación. XVII: 32 (2008 marzo) 29-48.

Joumard, Isabelle; André, Cristophe; Nicq, Chantal (2010). Health care systems: Efficiency and institutions // OECD Economics Department Working Papers No. 769. OECD Publishing, Paris. DOI: 10.787/5kmfp51f5f9t-en.

Klein, M. S.; Ross, F. V.; Adams, D. L.; Gilbert, C. M. (1994). Effect of online literature searching on length of stay and patient care costs // Academic Medicine. 69:6 489-95.

Marshall, J. G. (1992). The impact of the hospital library on clinical decision making: the Rochester study. // Bulletin of the Medical Library Association. 80:2 169-178.
Muñoz González, L.; Juan Quilis, V. (2011). Is a Virtual Library cost effective?. // Journal of European Association of Health Information Libraries. 7:4, 3-6. http://hdl.handle.net/10668/550 (2015-10-31).

National Health Service (2015). National Institute for Health and Care Excellence. http://www.nice.org.uk (2015-1010).

National Institutes of Health (2015). U.S. National Library of Medicine. Bethesda. http://www.nlm.nih.gov (2015-7-4)

Norwegian Electronic Health Library (2015). Norwegian Electronic Health Library. http://www.helsebiblioteket.no/ english (2015-10-22).

Nylenna, M.; Eiring, Ø.; Strand, G.; Røttingen, J. A. (2010). Wiring a nation: putting knowledge into action. // The Lancet. 375:9719 1048-1051.

Organisation for Economic Co-operation and Development (OECD). (2015). OECD Health Statistics 2015. http://stats.oecd.org (2016-2-25).

Packer, A. L. (2000). BIREME y el Sistema Latinoamericano y del Caribe de Información en Ciencias de la Salud. Hacia la Biblioteca Virtual en Salud. // Revista Medicina. 22:1 54-60.

Rajakiili, P. (2002). The national electronic library in Finland, FinElib. // Health Information \& Libraries Journal. 19:3 169-172.

Rodríguez Alonso, F. (1993). Hacia un Sistema Nacional de Información y Documentación en Salud: una propuesta de acción. $4^{\circ}$ Jornadas de Información y Documentación en Ciencias de la Salud. Bilbao, 16, 17 y 18 junio 1992. Vitoria: Asociación Vasca de Archiveros, Bibliotecarios y Documentalistas, 1993. 13-21.

Roqué Castella, Pilar; Tomé, María José.; Beceiro López, Gabriel; Armengol, Gemma; Caro, Conxi; Catalán Vega, Marcos Antonio; Pérez-Ventana Ortiz, Carme; Cusco Planas, Teresa; Viladot, Agustí. (2011). En busca de un modelo de red bibliotecaria: estudio comparativo de las redes de bibliotecas sanitarias y otros modelos de red. // Juan Quilis, Verónica (coord.) Bibliosalud 2011: XIV Jornadas Nacionales de Información y Documentación en Ciencias de la Salud, Cádiz 13-15 de abril de 2011. 7798.

Terkko. (2015). Terkko Navigator. https://www.terkko.helsin ki.fil (2015-10-6)

Tjensvoll, K. (2011). National licensing for the Norwegian Electronic Health Library // Serials. 24:1, 52-55.

Toth, B.; Muir Gray, J. A.; Fraser, V.; Ward, R. (2000). National electronic Library for Health: progress and prospects // Health Libraries Review. 17:1 46-50.

University of Helsinki. (2015). Finnish National Electronic Library, FinELib. http://www.nationallibrary.fi/en/libraries/ finelib.html (2015-10-10).

Enviado: 2016-03-17. Segunda versión: 2016-10-05 Aceptado: 2016-10-05. 